\title{
Association of Helicobacter pylori infection and environmental factors in non-cardia gastric cancer in Japan
}

\author{
Ai Machida-Montani ${ }^{1,2}$, Shizuka Sasazuki ${ }^{1}$, Manami Inoue ${ }^{1}$, Syusuke Natsukawa ${ }^{3}$, Kozo Shaura $^{4}$, \\ Yoichi Koizumi ${ }^{5}$, Yoshio Kasuga ${ }^{6}$, Tomoyuki Hanaoka ${ }^{1}$, and Shoichiro Tsugane ${ }^{1}$ \\ ${ }^{1}$ Epidemiology and Biostatistics Division, National Cancer Center Research Institute East, 6-5-1 Kashiwanoha, Kashiwa, \\ Chiba 277-8577, Japan \\ ${ }^{2}$ Department of Gastroenterology, Osaka City University Graduate School of Medicine, Osaka, Japan \\ ${ }^{3}$ Saku General Hospital, Nagano, Japan \\ ${ }^{4}$ Hokushin General Hospital, Nagano, Japan \\ ${ }^{5}$ Shinonoi General Hospital, Nagano, Japan \\ ${ }^{6}$ Nagano Matsushiro General Hospital, Nagano, Japan
}

\begin{abstract}
Background. Although Helicobacter pylori infection is a major risk factor for gastric cancer, it does not explain the full picture of stomach carcinogenesis. There have been few epidemiological studies, however, which examined both $\boldsymbol{H}$. pylori and environmental factors simultaneously. The aims of this study were to estimate the association of environmental factors (smoking and dietary factors) with gastric cancer in consideration of $\boldsymbol{H}$. pylori infection, and to investigate the effects of the interaction between environmental factors and $H$. pylori infection.

Methods. A multicenter, hospital-based, case-control study of gastric cancer was conducted at four hospitals in Nagano prefecture, Japan, between October 1998 and March 2002. For 153 newly diagnosed gastric cancer cases, two controls matched by age (within 3 years), sex, and residence area were randomly selected from the participants of a health check-up program during the same period in the same hospitals. We conducted a questionnaire survey and obtained blood samples. Consequently, 122 non-cardia gastric cancer cases and 235 controls were available for this analysis.

Results. H. pylori infection was strongly associated with noncardia gastric cancer after adjustment for possible confounding factors (odds ratio [OR], 8.2; 95\% confidence interval [CI], 3.7-18.2). Cigarette smoking (OR, 2.8; 95\% CI, 1.2-6.5) and frequent intake of miso (fermented soy bean) soup (OR, 2.1; 95\% CI, 0.9-5.1) and rice (OR, 2.5; 95\% CI, 1.0-6.1) were determined to be risk factors even after adjusting for possible confounding factors, including $\mathrm{H}$. pylori infection. However, no statistically significant interaction between environmental factors and $\boldsymbol{H}$. pylori infection was detected.

Conclusion. This finding suggests that although $H$. pylori infection is clearly an important risk factor for gastric cancer, smoking cessation and dietary modification may be practical strategies for the prevention of non-cardia gastric cancer
\end{abstract}

Offprint requests to: $\mathrm{S}$. Tsugane

Received: August 12, 2003 / Accepted: January 5, 2004 among both $H$. pylori-positive and -negative subjects in Japan.

Key words Helicobacter pylori - Gastric cancer · Smoking · Diet · Case-control study

\section{Introduction}

In 1994, the International Agency for Research on Cancer recognized Helicobacter pylori as a class I human carcinogen. A combined analysis of 12 case-control studies nested within prospective cohorts suggested that the odds ratio (OR) for the association between $H$. pylori infection and gastric cancer was 2.36 (95\% confidence intervals [CIs], 1.98-2.81) [1]. Thus, there is a strong link between $H$. pylori and gastric cancer in many countries. By contrast, low gastric cancer rates have been reported in some countries with a high prevalence of $H$. pylori infection, such as India and Bangladesh [2]. This difference in gastric cancer rates in populations with similar high prevalences of $H$. pylori infection could be related to the difference in the diversity of $H$. pylori strains, ethnicity, and environmental factors.

There is recently increasing evidence that $H$. pylori strains that possess the cytotoxin-associated gene A (CagA) are associated with an increased risk of atrophic gastritis and gastric cancer. CagA-positive H. pylori infection strongly increased the risk for gastric cancer compared with $H$. pylori-uninfected subjects [3-6].

In a case-control study in Brazil, Tatemichi et al. [7] showed there were ethnic differences in the strength of the association between CagA serological status and non-cardia gastric cancer, which suggested that environmental factors played an important role in gastric car- 
cinogenesis in Japanese Brazilians compared to nonJapanese Brazilians.

A relationship between environmental factors and gastric cancer has been reported in numerous previous studies [8-12]. A low intake of vegetables and a high intake of salt and salty foods were considered to be risk factors for gastric cancer [10,11]. Our previous study showed the protective effect of mushrooms and cruciferous vegetables among the same subjects as those of this present study, although we did not take into account $H$. pylori infection [12]. Moreover, there have been few epidemiological studies that have investigated the association between environmental factors and gastric cancer in consideration of $H$. pylori infection, and the interaction between environmental factors and $H$. pylori infection [13-16].

Concerning anatomical subsites, gastric cancer has been divided into two groups: "cardia" cancer (the upper third around the cardia) and "non-cardia" cancer (middle body and antrum). In the study by Hansen et al. [17], H. pylori infection was found to be associated with an increased risk of non-cardia cancer, but had an inverse association with cardia cancer. Our study subjects were restricted to cases of non-cardia cancer, which had a strong association with $H$. pylori infection.

The aim of this study was not only to confirm the association between non-cardia gastric cancer and $H$. pylori infection, combined with CagA, but also to estimate the association of environmental factors (smoking and dietary factors) with non-cardia cancer in consideration of $H$. pylori infection, which is a prevalent risk factor. We also investigated the effects of the interaction between environmental factors and $H$. pylori infection.

\section{Subjects and methods}

\section{Subjects}

A multicenter, hospital-based, case-control study of stomach cancer was conducted at four hospitals in Nagano prefecture, Japan, between October 1998 and March 2002. One hundred and fifty-three incident cases (in patients aged 20 to 74 years), of gastric cancer with histologic confirmation were newly diagnosed at these hospitals. For the 153 gastric cancer cases identified, two controls matched by age (within 3 years), sex, and residence area were randomly selected from the participants of a health check-up program during the same period in the same hospitals; they were confirmed to be cancer-free and with no past history of cancer. We conducted a questionnaire survey and obtained blood samples, with signed informed consent, from the 153 cancer-case patients and the 301 control subjects, before treatment for gastric cancer. Of the 153 gastric cancer cases, cardia cancer cases (in the upper third around the cardia), cases with uninformative anatomical subsite, and their control subjects were excluded from this study. A total of 126 non-cardia gastric cancer cases (middle body and antrum) and 247 controls remained. We also excluded subjects with extreme caloric intake (for men, $<500$ or $\geq 4000 \mathrm{kcal} / \mathrm{day}$; for women, $<400$ or $\geq 3500 \mathrm{kcal} /$ day), because this information was unreliable. Consequently, 122 non-cardia cancer cases and 235 controls were available for this analysis.

\section{Questionnaire}

The questionnaire was composed of items such as general characteristics (age, sex, sociodemographic characteristics), personal medical history, family history, smoking and drinking history, supplement use, and dietary factors. We also asked whether they were members of the Japan Agricultural Cooperatives (JA), because JA members may have particular dietary habits. JA members, who were farmers, were more familiar with health check-up programs than non-members. Therefore, we performed a careful multivariate adjustment for confounding factors, including JA membership, in order to exclude the effects of these factors.

All subjects were asked about the average frequency of intake and portion size of 141 items in the year preceding the interview or before a change of dietary habit, if the change had occurred in the past year. If they had any symptoms, their habitual intake prior to the symptoms was elicited. Daily consumption of rice and miso soup was classified into nine categories $(<1,1,2,3,4,5$, $6,7-9,10+$ cups/day). The frequency of other food items was classified into nine categories (never, 1-3 times/month, 1-2, 3-4, or 5-6 times/week, almost once/ day, 2-3, 4-6 or $>7$ times/day), and the portion size was classified into three categories (more than 1.5 portions, same as the usual portion size, or less than half a portion). The mean daily consumption of each food group was calculated by multiplying the frequency and portion size. The estimated intake calculated by the questionnaire had been validated against a 14- or 28-day dietary record in a prior study. The Spearman correlation coefficients for intake of total vegetables, total fruits, pickled vegetables, and sodium were $0.36,0.61,0.74$, and 0.59 in males and $0.34,0.50,0.75$, and 0.55 in females, respectively $[18,19]$.

\section{Laboratory data}

The study subjects were tested for serum pepsinogen I (PG I) and pepsinogen II (PG II), and for IgG antibody to H. pylori ( $\mathrm{Hp}-\mathrm{Ab})$ and $\mathrm{CagA}(\mathrm{CagA}-\mathrm{Ab})$. These antibodies were measured with an enzyme-linked 
immunosorbent assay (ELISA; Helico G; PortonCambridge, Oxford, UK) kit and CagA kit (RADIM, Rome, Italy). Equal to and more than ten units per milliliter $(\mathrm{U} / \mathrm{ml})$ were considered a positive test in both $\mathrm{Hp}-\mathrm{Ab}$ and CagA-Ab. H. pylori infection was defined when one or both serum assays were positive. The serum PG I and PG II levels were measured by radioimmunometric assay kits (PG1/PG2 RIABEAD; Dainabot, Tokyo, Japan). Atrophic gastritis was diagnosed according to the criteria of a PG I level below $70 \mathrm{ng} / \mathrm{ml}$ and a PG I/PG II ratio below 3.0. The prevalence of serologically diagnosed atrophic gastritis using these criteria was well correlated $(r=0.999 ; P<0.0001)$ with the age-adjusted mortality rate of gastric cancer among five Japanese populations [20].

To estimate the association between environmental factors and non-cardia cancer, the total intake of each food group and the frequency of each food item were each divided into three categories, at the nearest tertile based on the distribution in the control group. Smoking status was classified as never, past, or current smoker. The linear trend was assessed by assigning ordinal values for categorical factors.

In order to estimate the joint effects of environmental factors and $H$. pylori infection, the total consumption of vegetables, fruits, and salt was classified into a highintake (above median intake) or a low-intake group (below median intake) according to the distribution of the control group. The number of cups per day was classified in two categories (high and low intake) for miso soup and rice. Smoking status was classified into two groups: never smoker and ever (past or current) smoker. We assessed the association with non-cardia cancer by taking into consideration the patterns of joint occurrence of both factors (environmental factors and $H$. pylori infection). Furthermore, the effect of interaction was checked by calculating an interaction term, and multiplying a dummy variable for each environmental factor by one for $H$. pylori infection.
Odds ratios (ORs) and 95\% confidence intervals (95\% CIs) and trends were obtained by conditional logistic regression analysis. All $P$ values were two-sided and all the statistical analysis was performed using the SAS statistical software package [21].

\section{Results}

The mean ages of cases and controls were 57.8 and 57.4 years, respectively. The proportion of male participants was $67.2 \%$ in cases and $67.7 \%$ in controls.

Table 1 shows the crude and adjusted ORs of $H$. pylori combined with CagA for non-cardia cancer. The crude OR of $H$. pylori infection for non-cardia cancer was 7.0 (95\% CI, 3.3-14.8). The highest OR was observed in the H. pylori seropositivity and CagA seropositivity $[\mathrm{Hp}(+) \mathrm{CagA}(+)]$ category (OR, 10.1; 95\% CI, 4.4-23.1). The H. pylori seropositivity and CagA seronegativity $[\mathrm{Hp}(+) \mathrm{CagA}(-)]$ category had an estimated OR of 2.4 , but this was not statistically significant. After adjustment for confounding variables, the ORs of $H$. pylori and/or CagA with gastric cancer increased.

Table 2 shows the association of environmental factors with the risk of non-cardia cancer. As for smoking, crude ORs for past and current smoking were 3.3 (95\% CI, 1.6-6.7) and 2.8 (95\% CI, 1.3-5.8), respectively. Past and current smoking remained, in the multivariate analysis, significantly associated with non-cardia cancer. Regarding salt intake, we found a slightly elevated OR (for high-intake category, OR, 1.5; 95\% CI, 0.6-3.7), which did not reach statistical significance. As the consumption of miso soup increased, we found a significantly increased OR (for high-intake category, OR, 2.1; 95\% CI, 0.9-5.1; $P$ for trend $=0.04)$. A marginal positive association between rice intake and non-cardia cancer was observed (for the high-intake category, OR, 2.5; 95\% CI, 1.0-6.1; $P$ for trend $=0.07$ ).

Table 1. Crude and adjusted odds ratios (ORs) and $95 \%$ confidence interval (CIs) ${ }^{\mathrm{a}}$ of Helicobacter pylori infection for non-cardia cancer

\begin{tabular}{lcccccr}
\hline & $\begin{array}{c}\text { Cases } \\
(n=122)\end{array}$ & $\begin{array}{c}\text { Controls } \\
(n=235)\end{array}$ & $\begin{array}{c}\text { Crude } \\
\text { OR }\end{array}$ & $\begin{array}{c}\text { 95\% CI } \\
\text { Adjusted } \\
\text { OR }^{\mathrm{b}}\end{array}$ & $95 \%$ CI \\
\hline H. pylori infection combined with CagA & & & & & & \\
H. pylori (-) and CagA (-) & 10 & 84 & 1.0 & & 1.0 & \\
H. pylori (+) or CagA (+) & 112 & 151 & 7.0 & $3.3-14.8$ & 8.2 & $3.7-18.2$ \\
H. pylori (-) and CagA (+) & 9 & 17 & 5.3 & $1.7-16.0$ & 6.0 & $1.8-19.8$ \\
H. pylori (+) and CagA (-) & 8 & 27 & 2.4 & $0.8-6.9$ & 2.5 & $0.8-7.4$ \\
H. pylori (+) and CagA (+) & 95 & 107 & 10.1 & $4.4-23.1$ & 13.4 & $5.4-33.3$ \\
\hline
\end{tabular}

H. pylori infection was defined when one or both serum assays (H. pylori and CagA) were positive

JA, Japan Agricultural Cooperatives

${ }^{a}$ Conditional logistic regression analysis

${ }^{\mathrm{b}}$ Adjusted for smoking status, JA membership, family history of gastric cancer, total vegetable intake, total fruits intake, and salt intake 
Table 2. Crude and adjusted odds ratios (ORs) and $95 \%$ confidence intervals (CIs) a of environmental factors for non-cardia cancer

\begin{tabular}{|c|c|c|c|c|c|c|c|c|}
\hline & $\begin{array}{c}\text { Cases } \\
(n=122)\end{array}$ & Percentage & $\begin{array}{c}\text { Controls } \\
(n=235)\end{array}$ & Percentage & $\begin{array}{c}\text { Crude } \\
\text { OR }\end{array}$ & $95 \% \mathrm{CI}$ & $\begin{array}{l}\text { Adjusted } \\
\text { OR }\end{array}$ & $95 \% \mathrm{CI}$ \\
\hline \multicolumn{9}{|l|}{ Smoking ${ }^{b}$} \\
\hline Never & 44 & $(36.1)$ & 119 & $(50.6)$ & 1.0 & & 1.0 & \\
\hline Past & 39 & $(32.0)$ & 53 & $(22.6)$ & 3.3 & $1.6-6.7$ & 2.8 & $1.3-6.3$ \\
\hline Current & 39 & $(32.0)$ & 63 & $(26.8)$ & 2.8 & $\begin{array}{c}1.3-5.8 \\
0.01\end{array}$ & 2.8 & $\begin{array}{c}1.2-6.5 \\
0.03\end{array}$ \\
\hline \multicolumn{9}{|l|}{ Salt ${ }^{c}$} \\
\hline Tertile 1 (8.7) & 46 & $(37.7)$ & 78 & $(33.2)$ & 1.0 & & 1.0 & \\
\hline Tertile 2 (13.0) & 33 & $(27.0)$ & 78 & $(33.2)$ & 0.8 & $0.5-1.3$ & 1.3 & $0.7-2.7$ \\
\hline Tertile 3 (20.4) & 43 & $(35.2)$ & 79 & $(33.6)$ & 0.9 & $\begin{array}{c}0.6-1.5 \\
0.73\end{array}$ & 1.5 & $\begin{array}{c}0.6-3.7 \\
0.36\end{array}$ \\
\hline \multicolumn{9}{|l|}{ Miso soup ${ }^{\mathrm{d}}$} \\
\hline$<3$ cups/day & 47 & $(38.5)$ & 100 & $(42.6)$ & 1.0 & & 1.0 & \\
\hline 3 cups/day & 54 & (44.3) & 99 & $(42.1)$ & 1.2 & $0.7-1.9$ & 1.8 & $1.0-3.3$ \\
\hline$\geqq 4$ cups/day & 21 & $(17.2)$ & 36 & $(15.3)$ & 1.2 & $\begin{array}{c}0.6-2.4 \\
0.48\end{array}$ & 2.1 & $\begin{array}{c}0.9-5.1 \\
0.04\end{array}$ \\
\hline \multicolumn{9}{|l|}{ Pickled vegetables ${ }^{\mathrm{d}}$} \\
\hline Tertile 1 (10.9) & 52 & $(42.6)$ & 79 & $(33.6)$ & 1.0 & & 1.0 & \\
\hline Tertile 2 (36.6) & 33 & $(27.0)$ & 78 & $(33.2)$ & 0.7 & $0.4-1.1$ & 0.6 & $0.3-1.2$ \\
\hline Tertile 3 (79.8) & 37 & $(30.3)$ & 78 & $(33.2)$ & 0.7 & $\begin{array}{c}0.4-1.2 \\
0.22\end{array}$ & 0.6 & $\begin{array}{c}0.3-1.3 \\
0.17\end{array}$ \\
\hline \multicolumn{9}{|l|}{ Rice $^{d}$} \\
\hline$<4$ cups/day & 31 & $(25.4)$ & 62 & $(26.4)$ & 1.0 & & 1.0 & \\
\hline 4 cups/day & 64 & $(52.5)$ & 129 & $(54.9)$ & 1.1 & $0.6-1.8$ & 1.2 & $0.6-2.3$ \\
\hline$\geqq 5 \mathrm{cups} /$ day & 27 & $(22.1)$ & 44 & $(18.7)$ & 1.4 & $\begin{array}{c}0.7-2.8 \\
0.39\end{array}$ & 2.5 & $\begin{array}{c}1.0-6.1 \\
0.07\end{array}$ \\
\hline \multicolumn{9}{|l|}{ Total vegetable } \\
\hline Tertile 1 (119.2) & 50 & $(41.0)$ & 79 & $(33.6)$ & 1.0 & & 1.0 & \\
\hline Tertile 2 (218.4) & 33 & $(27.0)$ & 78 & (33.2) & 0.7 & $0.4-1.2$ & 0.7 & $0.3-1.4$ \\
\hline Tertile 3 (377.0) & 39 & $(32.0)$ & 78 & $(33.2)$ & 0.8 & $\begin{array}{c}0.5-1.3 \\
0.34\end{array}$ & 0.9 & $\begin{array}{c}0.4-2.2 \\
0.83\end{array}$ \\
\hline \multicolumn{9}{|l|}{ Total fruit ${ }^{\mathrm{f}}$} \\
\hline Tertile 1 (50.7) & 44 & $(36.1)$ & 77 & $(32.8)$ & 1.0 & & 1.0 & \\
\hline Tertile $2(142.5)$ & 43 & $(35.2)$ & 80 & (34.0) & 1.0 & $0.6-1.6$ & 1.4 & $0.7-2.6$ \\
\hline Tertile 3 (294.0) & 35 & $(28.7)$ & 78 & $(33.2)$ & 0.8 & $\begin{array}{c}0.4-1.3 \\
0.36\end{array}$ & 1.1 & $\begin{array}{c}0.5-2.4 \\
0.73\end{array}$ \\
\hline
\end{tabular}

Tertile 1 , lowest tertile; tertile 2 , intermediate tertile; tertile 3 , highest tertile (median intake)

${ }^{a}$ Conditional logistic regression analysis

${ }^{\mathrm{b}}$ Adjusted for $\mathrm{H}$. pylori infection, JA membership, family history of gastric cancer, total vegetable intake, total fruit intake, and salt intake

'Adjusted for $\mathrm{H}$. pylori infection, smoking status, JA membership, family history of gastric cancer, total vegetable intake, total fruit intake, and total energy intake

${ }^{\mathrm{d}}$ Adjusted for H. pylori infection, smoking status, JA membership, family history of gastric cancer, total vegetable intake, total fruit intake, salt intake, and total energy intake

${ }^{\mathrm{e}}$ Adjusted for $\mathrm{H}$. pylori infection, smoking status, JA membership, family history of gastric cancer, total fruit intake, salt intake, and total energy intake

${ }^{\mathrm{f}}$ Adjusted for $\mathrm{H}$. pylori infection, smoking status, JA membership, family history of gastric cancer, total vegetable intake, salt intake, and total energy intake

High intake of pickled vegetables and low intake of total vegetables and fruits was not associated with noncardia cancer.

The joint effects of environmental factors (smoking and foods) and H. pylori infection on the risk for noncardia cancer are presented in Table 3. As for smoking status and H. pylori infection, the adjusted OR for noncardia cancer was 1.9 (95\% CI, 0.4-8.8) for the $H$. pylori-negative smoker group, 6.4 (95\% CI, 2.1-19.7) for the H. pylori-positive never smoker group, and 19.0
(95\% CI, 5.4-67.2) for the H. pylori-positive smoker group. An H. pylori-positive smoker had 3.0 (95\% CI, 1.4-6.6) times the risk of non-cardia cancer compared to an $H$. pylori-positive never-smoker (data not shown in Table 3).

For salt, miso soup, pickled vegetables and rice, the ORs were compared with the $H$. pylori-negative subjects with lower intake as a reference. As for salt intake and $H$. pylori infection, the adjusted OR for gastric cancer was 1.8 (95\% CI, 0.4-7.7) for H. pylori-negative 
Table 3. Joint effect of environmental factors and H. pylori infection on the risk for non-cardia cancer

\begin{tabular}{|c|c|c|c|c|c|c|c|}
\hline & $\begin{array}{c}\text { Cases } \\
(n=122)\end{array}$ & $\begin{array}{l}\text { Controls } \\
(n=235)\end{array}$ & $\begin{array}{l}\text { Crude } \\
\text { OR }\end{array}$ & $95 \%$ CI & $\begin{array}{l}\text { Adjusted } \\
\text { OR }\end{array}$ & $95 \%$ CI & $\begin{array}{c}\text { Interaction } \\
\text { term }\end{array}$ \\
\hline \multicolumn{8}{|l|}{ Smoking ${ }^{a}$} \\
\hline H. pylori $(-)$ and never smoking & 4 & 43 & 1.0 & & 1.0 & & \\
\hline H. pylori $(-)$ and smoking & 6 & 41 & 2.0 & $0.5-9.1$ & 1.9 & $0.4-8.8$ & \\
\hline H. pylori $(+)$ and never smoking & 40 & 76 & 5.3 & $1.8-15.8$ & 6.4 & $2.1-19.7$ & \\
\hline H. pylori $(+)$ and smoking & 72 & 75 & 17.7 & $5.2-60.5$ & 19.0 & $5.4-67.2$ & 0.52 \\
\hline \multicolumn{8}{|l|}{ Salt $^{\mathrm{b}}$} \\
\hline H. pylori $(-)$ and low intake & 4 & 41 & 1.0 & & 1.0 & & \\
\hline H. pylori $(-)$ and high intake & 6 & 43 & 1.5 & $0.4-5.9$ & 1.8 & $0.4-7.7$ & \\
\hline H. pylori $(+)$ and low intake & 58 & 76 & 8.9 & $2.8-27.6$ & 9.7 & $3.0-31.7$ & \\
\hline H. pylori $(+)$ and high intake & 54 & 74 & 9.0 & $2.8-28.3$ & 14.2 & $3.9-52.3$ & 0.56 \\
\hline \multicolumn{8}{|l|}{ Miso soup ${ }^{c}$} \\
\hline H. pylori $(-)$ and low intake & 8 & 77 & 1.0 & & 1.0 & & \\
\hline H. pylori $(-)$ and high intake & 2 & 7 & 2.1 & $0.3-13.7$ & 3.0 & $0.4-24.1$ & \\
\hline H. pylori $(+)$ and low intake & 93 & 122 & 7.6 & $3.4-17.0$ & 9.0 & $3.8-21.3$ & \\
\hline H. pylori $(+)$ and high intake & 19 & 29 & 8.4 & $3.0-23.4$ & 12.6 & $4.0-39.4$ & 0.52 \\
\hline \multicolumn{8}{|l|}{ Pickled vegetables ${ }^{\mathrm{c}}$} \\
\hline H. pylori $(-)$ and low intake & 6 & 45 & 1.0 & & 1.0 & & \\
\hline H. pylori $(-)$ and high intake & 4 & 39 & 0.9 & $0.2-3.5$ & 0.7 & $0.2-3.3$ & \\
\hline H. pylori $(+)$ and low intake & 63 & 73 & 7.4 & $2.7-20.2$ & 8.0 & $2.8-22.8$ & \\
\hline H. pylori $(+)$ and high intake & 49 & 78 & 5.7 & $2.1-15.7$ & 6.4 & $2.0-19.9$ & 0.84 \\
\hline \multicolumn{8}{|l|}{ Rice $^{\mathrm{c}}$} \\
\hline H. pylori $(-)$ and low intake & 8 & 63 & 1.0 & & 1.0 & & \\
\hline H. pylori $(-)$ and high intake & 2 & 21 & 0.7 & $0.1-3.9$ & 0.9 & $0.2-5.4$ & \\
\hline H. pylori $(+)$ and low intake & 87 & 128 & 5.8 & $2.5-13.5$ & 7.3 & $2.9-18.4$ & \\
\hline H. pylori $(+)$ and high intake & 25 & 23 & 11.0 & $3.9-30.9$ & 18.7 & $5.6-62.6$ & 0.31 \\
\hline \multicolumn{8}{|l|}{ Total vegetable } \\
\hline H. pylori $(-)$ and high intake & 4 & 36 & 1.0 & & 1.0 & & \\
\hline H. pylori $(-)$ and low intake & 6 & 48 & 0.9 & $0.2-3.6$ & 1.0 & $0.2-4.4$ & \\
\hline H. pylori $(+)$ and high intake & 48 & 81 & 5.5 & $1.8-16.9$ & 7.6 & $2.3-25.2$ & \\
\hline H. pylori $(+)$ and low intake & 64 & 70 & 7.5 & $2.5-22.3$ & 8.5 & $2.4-29.9$ & 0.60 \\
\hline \multicolumn{8}{|l|}{ Total fruit ${ }^{\mathrm{e}}$} \\
\hline H. pylori $(-)$ and high intake & 5 & 39 & 1.0 & & 1.0 & & \\
\hline H. pylori $(-)$ and low intake & 5 & 45 & 0.8 & $0.2-3.2$ & 0.9 & $0.2-3.9$ & \\
\hline H. pylori $(+)$ and high intake & 44 & 78 & 4.8 & $1.7-13.0$ & 5.8 & $2.0-16.9$ & \\
\hline H. pylori $(+)$ and low intake & 68 & 73 & 7.9 & $2.8-21.8$ & 10.6 & $3.3-33.9$ & 0.32 \\
\hline
\end{tabular}

Salt, pickled vegetable, total vegetable and fruit ; low (below median) intake, high (above median) intake

Miso soup; low intake ( $<3$ cups/day), high intake ( $\geqq 3$ cups/day)

Rice; low intake ( $<4$ cups/day), high intake ( $\geqq 4$ cups/day)

H. pylori infection was defined when one or both serum assays (H. pylori and CagA) were positive

${ }^{a}$ Adjusted for JA membership, family history of gastric cancer, total vegetable intake, total fruit intake, and salt intake

${ }^{\mathrm{b}}$ Adjusted for JA membership, smoking status, family history of gastric cancer, total vegetable intake, total fruit intake, and total energy intake ${ }^{\mathrm{c}}$ Adjusted for JA membership, smoking status, family history of gastric cancer, total vegetable intake, total fruit intake, salt intake and total energy intake

${ }^{\mathrm{d}}$ Adjusted for JA membership, smoking status, family history of gastric cancer, total fruit intake, salt intake, and total energy intake

${ }^{\mathrm{e}}$ Adjusted for JA membership, smoking status, family history of gastric cancer, total vegetable intake, salt intake, and total energy intake

subjects with a high intake, 9.7 (95\% CI, 3.0-31.7) for $H$. pylori-positive subjects with a low intake, and $14.2(95 \%$ CI, 3.9-52.3) for H. pylori-positive subjects with a high intake. After adjustment for confounding factors including smoking status, high intake of miso soup tended to increase the ORs of non-cardia cancer in both $H$. pylori-positive and -negative subjects. Regarding rice consumption, the adjusted OR was 7.3 (95\% CI, 2.918.4) for $H$. pylori-positive subjects with a low intake and 18.7 (95\% CI, 5.6-62.6) for H. pylori-positive subjects with a high intake. High rice consumption was not associated with non-cardia cancer among H. pylorinegative subjects.
ORs were compared with $H$. pylori-negative subjects with a high intake of total vegetables and total fruits as the reference. Low intake of vegetables/fruits did not increase the risk compared with high intake among $H$. pylori-negative subjects. However, a marginal positive association between low intake of total fruits and noncardia cancer was observed among $H$. pylori-positive subjects.

Additionally, no significant improvement in the regression model was observed when the interaction term between these environmental factors (smoking, food groups, and food items) and H. pylori infection was added. 


\section{Discussion}

In this study, $H$. pylori infection was defined as present when one or both serum assays were positive. Clinical data indicated that the CagA antibody persisted longer after eradication treatment than the antibody detected by $H$. pylori $\mathrm{IgG}$ [22]. It seems reasonable to assume that the addition of a test for the CagA antibody will result in a more correct representation of past exposure than the use of the $H$. pylori $\mathrm{IgG}$ antibody alone, as Ekstrom et al. [23] suggested.

We confirmed the strong association between $H$. pylori infection and non-cardia cancer. In this study, a significantly increased risk was observed in the $H$. pylori seropositivity and CagA seropositivity $[\mathrm{Hp}(+) \mathrm{CagA}(+)]$ category, while risk was moderately but not significantly increased between the $H$. pylori seropositivity and CagA seronegativity category. This suggested that CagA-positive H. pylori infection had strongly increased the risk for non-cardia cancer compared with $H$. pylori-negative subjects, consistent with previous studies [3-6].

As for smoking status, smoking tended to increase the risk for gastric cancer irrespective of $H$. pylori infection. H. pylori-positive smokers showed the highest OR, of 19.0, compared to $H$. pylori-negative never-smokers. Smoking proved to be a risk factor even among subjects with $H$. pylori infection. Some mechanisms of smoking associated with the increased risk of gastric cancer have been suggested. Smokers show lower plasma levels of antioxidants such as vitamin C and beta-carotene [24], and tobacco smoke contains carcinogenic nitrosamines, triggering the carcinogenesis of gastric carcinoma [25]. An increased risk of gastric cancer from smoking was observed in two prospective studies [26,27]. From investigating the joint effects of $H$. pylori and smoking status, Siman et al. [28] suggested that smoking and $H$. pylori were both risk factors for gastric cancer, and that smoking was still a risk factor among $H$. pylori-positive individuals. Brenner et al. [16] found that CagA-positive smokers had an increased risk of non-cardia gastric cancer in a close to multiplicative way, leading to a 16.6-fold risk increase compared with non-smokers without $H$. pylori infection. In a Russian case-control study, smoking had no effect on the risk of gastric cancer in $H$. pylori-negative men, but it was associated with a significantly increased risk in $H$. pylori-positive men ( $P$ value for interaction, 0.07) [29].

Excessive salt intake is a risk factor supported by experimental and epidemiological studies [10,30,31]. The promoting effects of salt may be caused through a mechanism by which high salt concentrations destroy the mucosal barrier that protects the surface membrane of the stomach [32]. In Mongolian gerbils, the synergistic promoting effects of $H$. pylori infection and a high- salt diet (containing 10\% sodium chloride) on gastric carcinogenesis were observed [31]. In the present study, although high salt intake caused a slightly increased OR, it was not statistically significant. In the analysis of joint effects, $H$. pylori infection with a high salt intake had an elevated OR for non-cardia cancer compared with the reference category, but failed to show a statistically significant increase in ORs compared with $H$. pylori-positive subjects with a low intake (data not shown). Part of the reason for this result was the difficulty in estimating the exact amount of salt consumption from the questionnaire. Moreover, we may find a synergistic promoting effect of salt intake on gastric cancer only in subjects who consistently consume high levels of salt.

As miso soup and rice intake increased, we found a steady increase in the risk of non-cardia cancer. Because miso soup is one of the major contributors to sodium intake, this result is plausible. For rice, a marginal positive association was observed. A high rice intake significantly increased the risk (OR, 2.6; 95\% CI, 1.1-6.0) compared to low intake even in H. pyloripositive subjects. A positive association between high rice intake and $H$. pylori infection has been observed in some epidemiological studies [8,9]. Although a previous study suggested that carbohydrates irritated the gastric mucosa [33], the direct effect of rice on carcinogenesis in the stomach was unclear. As an indirect effect, there is a possibility that rice intake is a marker of salt, salty food, and other dietary factors that are risk factors for gastric cancer.

Vegetables and fruits contain many compounds with anticarcinogenic properties, including carotenoids and vitamin $\mathrm{C}$, and have been considered to be factors that protect against stomach carcinogenesis [10,11]. Our previous study showed the protective effect of mushrooms and cruciferous vegetables on the risk of gastric cancer among the same subjects as those in this present study [12]. The high consumption of total vegetables and fruits did not decrease the risk of non-cardia cancer after adjustment for $H$. pylori infection in our study. In the analysis of $H$. pylori infection and vegetables/fruits, low intake did not show an increased risk for non-cardia cancer compared with $H$. pylori-positive subjects with high intake, although $H$. pylori-positive subjects with low intake of total fruits tended to have an increased risk compared with $H$. pylori-positive subjects with high intake. In a previous study using the same food frequency questionnaire (FFQ), the average intake of vegetables and fruits was $215 \mathrm{~g} /$ day and $132 \mathrm{~g} /$ day among 18399 men, and $256 \mathrm{~g} /$ day and $202 \mathrm{~g} /$ day among 20932 women [34]. The lack of association could also be explained by a relatively high intake of these items in the two municipalities of Nagano prefecture comprising the present study area compared with that in other areas 
[35]. Kobayashi et al. [36], in a prospective study, suggested that vegetable and fruit intake, even in low amounts, was associated with a decreased risk; however, the risk did not decrease in a stepwise manner as the consumption increased. Therefore the decreased risk may have not been observed in the present study because these study subjects consumed relatively high amounts of vegetables and fruits. Another explanation may be that the protective effect of vegetable consumption, if any, may be modified or masked by other prevalent risk factors, for example, $H$. pylori infection.

The Spearman correlation coefficient for total vegetable intake assessed with FFQ and 14- or 28-day dietary records was relatively low. However, the intake assessed with FFQ was comparable to that with the 14or 28 days dietary record in the categorization of vegetable intake [18]. Moreover, we had observed an inverse association between vegetable intake and the risk of colorectal cancer in a simultaneously conducted casecontrol study using the same FFQ [12]. It is, therefore, unlikely that the failure to observe a protective association was due to the relatively low validity of the vegetable intake assessment.

In this study, there was almost no improvement at all in the logistic regression model when the interaction term between these environmental factors and $H$. pylori infection was added. The limited number of cases prohibited a more comprehensive assessment of gastric cancer risk according to the joint classification of subjects by environmental factors and $H$. pylori infection, and led to quite wide confidence intervals for some of the risk estimates. In other words, we might have failed to detect a statistically significant interaction owing to the small number of $H$. pylori-negative cases. Moreover, the high prevalence of $H$. pylori infection and its strong link to gastric cancer may have made precise evaluation of the association between environmental factors and gastric cancer virtually impossible.

There were some limitations in this study. First, the dietary information we collected was about the diet 1 year before the diagnosis. Such dietary information may not actually reflect past dietary habits, which may be more important for carcinogenesis in the stomach. Additionally, one of the major problems of case-control studies is the possibility of recall bias due to knowledge of the disease status. Information on food intake was collected after subsequent diagnosis of gastric cancer, so we could not avoid the recall bias inherent in casecontrol studies. Furthermore, cases might have changed their dietary habits as a consequence of gastric cancer and atrophic gastritis. Thus, dietary assessment is subject to considerable misclassification. In addition, the diagnosis of $\mathrm{H}$. pylori infection (including CagA) was based on immunological tests. The possibility of misclassification of $H$. pylori and CagA seropositivity by immunological tests was not excluded. However, if the misclassification occurred equally among all subjects, the risk of infection linked to the development of gastric cancer was underestimated. Another limitation of casecontrol studies is their potential selection bias. Among the controls in this study, the percentage of neversmokers was high because of the low number of female smokers. The percentages of past smokers and current smokers were $31.5 \%$ and $39.0 \%$, respectively, in the male controls. The percentage of current male smokers in this study was low compared with that in a population-based prospective study of 19576 Japanese men aged $40-59$ years $(53.4 \%)$ [26]. The low prevalence of smokers in the control group may have led to an overestimation of OR regarding cigarette smoking. Because the controls were participants in a medical health check-up program, they were considered to be more health-conscious than the general population. In addition, the JA members were more familiar with health check-up programs than non-members. Thus, we performed careful multivariate adjustment for confounding factors, including JA membership, as mentioned above.

We confirmed the strong association of $H$. pylori combined with CagA with non-cardia cancer. Regarding environmental factors, smoking and high intake of miso soup were associated with non-cardia cancer, regardless of $H$. pylori infection. Although a high rice intake and low fruit intake increased risk only among $H$. pylori-positive subjects, the interaction term was not statistically significant. However, we could not deny the interaction between $H$. pylori infection and environmental factors. Among the various factors studied here, $H$. pylori infection was shown to be the most important factor for non-cardia gastric cancer, indicating that eradication therapy for $H$. pylori would possibly be an effective strategy for reducing the risk of gastric cancer. Our study also indicates that other realistic alternatives may be smoking cessation and dietary modification for non-cardia gastric cancer, irrespective of $H$. pylori status. Further studies in a large number of subjects will be necessary to clarify the interaction between the various risk factors.

Acknowldgments The authors gratefully acknowledge the generous assistance of the staff members of each hospital and the Agricultural Technology Institute of the Nagano Farmers' Fenderation, Ms. Aoki, Mr. Ueki, Ms. Kimijima, Ms. Komatsu, Mr. Shimazaki, Ms. Horano, Mr. Yajima, Dr. Ikegawa, and Dr. Matsuzawa, and the laboratory staff member, Ms. Hashimoto. This study was supported in part by the Agricultural Technology Institute of the Nagano Farmers' Federation, a Grant-in-Aid for Cancer Research and the Second Term Comprehensive 10-year Strategy for Cancer Control from the Ministry of Health and Welfare of Japan, 
and a Grant-in-Aid for Scientific Research from the Ministry of Education, Culture, Sports, Science, and Technology, Japan.

\section{References}

1. Helicobacter and Cancer Collaborative Group. Gastric cancer and Helicobacter pylori: a combined analysis of 12 case control studies nested within prospective cohort. Gut 2001;49:347-53.

2. Miwa H, Go MF, Sato N. H. pylori and gastric cancer: the Asian enigma. Am J Gastroenterol 2002;97:1106-12.

3. Kuipers EJ, Perez-Perez GI, Meuwissen SGM, Blaser MJ. Helicobacter pylori and atrophic gastritis: importance of the CagA status. J Natl Cancer Inst 1995;87:1777-80.

4. Blaser MJ, Perez-Perez GI, Kleanthous H, Cover TL, Peek RM, Chyou PH, et al. Infection with Helicobacter pylori strains possessing cagA is associated with an increased risk of developing adenocarcinoma of the stomach. Cancer Res 1995;55:2111-5.

5. Parsonnet J, Friedman GD, Orentreich N, Vogelman H. Risk for gastric cancer in people with CagA positive or CagA negative Helicobacter pylori infection. Gut 1997;40:297-301.

6. Shimoyama T, Fukuda S, Tanaka M, Mikami T, Munakata A, Crabtree JE. CagA seropositivity associated with development of gastric cancer in Japanese population. J Clin Pathol 1998;51:2258.

7. Tatemichi M, Hamada GS, Nishimoto IN, Kowalski LP, Iriya K, Rodrigues JJG, et al. Ethnic difference in serology of Helicobacter pylori CagA between Japanese and non-Japanese Brazilians for non-cardia gastric cancer. Cancer Sci 2003;94:64-9.

8. Mathew A, Gangadharan P, Varghese C, Nair MK. Diet and stomach cancer: a case-control study in South India. Eur J Cancer Prev 2000;9:89-97.

9. Watabe K, Nishi M, Miyake H, Hirata K. Lifestyle and gastric cancer: a case-control study. Oncol Rep 1998;5:1191-4.

10. Ramon JM, Serra L, Cerdo C, Oromi J. Dietary factors and gastric cancer risk. A case-control study in Spain. Cancer 1993;71: 1731-5.

11. Buiatti E, Palli D, Bianchi S, Decarli A, Amadori D, Avellini C, et al. A case-control study of gastric cancer and diet in Italy. III. Risk patterns by histologic type. Int J Cancer 1991;48:369-74.

12. Hara M, Hanaoka T, Kobayashi M, Otani T, Adachi HY, Montani A, et al. Cruciferous vegetables, mushrooms, and gastrointestinal cancer risks in a multi-center, hospital-based case control study in Japan. Nutr Cancer 2003;46:138-47.

13. Zhang ZF, Kurtz RC, Klimstra DS, Yu GP, Sun M, Harlap S, et al. Helicobacter pylori infection on the risk of stomach cancer and chronic atrophic gastritis. Cancer Detect Prev 1999;5:357-67.

14. Wu AH, Crabtree JE, Bernstein L, Hawtin P, Cockburn M, Tseng $\mathrm{CC}$, et al. Role of Helicobacter pylori CagA + strains and risk of adenocarcinoma of the stomach and esophagus. Int $\mathbf{J}$ Cancer 2003;6:815-21.

15. Serafini M, Bellocco R, Wolk A, Ekstrom AM. Total antioxidant potential of fruit and vegetables and risk of gastric cancer. Gastroenterology 2002;123:985-91.

16. Brenner H, Arndt V, Bode G, Stegmaier C, Ziegler H, Stumer T. Risk of gastric cancer among smokers infected with Helicobacter pylori. Int J Cancer 2002;98;446-9.

17. Hansen S, Melby KK, Aase S, Jellium E, Vollset SE. Helicobacter pylori infection and risk of cardia cancer and non-cardia cancer. Scand J Gastroenterol 1999;4;353-60.

18. Sasaki S, Kobayashi M, Tsugane S. Validity of a self-administered food frequency questionnaire used in the 5-year follow-up survey of the JPHC Study Cohort I: comparison with dietary records for food groups. J Epidemiol 2003;13:S57-63.
19. Tsugane S, Kobayashi M, Sasaki S. Validity of the selfadministered food frequency questionnaire used in the 5-year follow-up survey of the JPHC study Cohort I: comparison with dietary records for main nutrients. J Epidemiol 2003;13:S51-6.

20. Kabuto M, Imai H, Tsugane S, Watanabe S. Correlation between atrophic gastritis prevalence and gastric cancer mortality among middle-aged men in five areas in Japan. J Epidemiol 1993;3:35-9.

21. SAS Institute. SAS user's guide. Cary, NC: SAS Institute; 1990.

22. Sorberg M, Engstrand L, Strom M, Jonsson KA, Jorbeck H, Granstrom $M$. The diagnostic value of enzyme immunoassay and immunoblot in monitoring eradication of Helicobacter pylori. Scand J Infect Dis 1997;2:147-51.

23. Ekstrom AM, Held M, Hansson LE, Engstrand L, Nyren O. Helicobacter pylori in gastric cancer established by CagA immunoblot as a marker of past infection. Gastroenterology 2001;121:784-91.

24. Buiatti E, Munoz N, Kato I, Vivas J, Muggli R, Plummer M, et al. Determinants of plasma anti-oxidant vitamin levels in a population at high risk for stomach cancer. Int J Cancer 1996;65:317-22.

25. Mirvish SS. Role of N-nitroso compounds (NOC) and Nnitrosation in etiology of gastric, esophageal and bladder cancer and contribution to cancer of known exposures to NOC. Cancer Lett 1995;1:17-48.

26. Sasazuki S, Sasaki S, Tsugane S. Cigarette smoking, alcohol consumption and subsequent gastric cancer risk by subsite and histologic type. Int J Cancer 2002;101:560-6.

27. Nomura A, Grove JS, Stemmermann GN, Severson RK. A prospective study of stomach cancer and its relation to diet, cigarettes, and alcohol consumption. Cancer Res 1990;3:62731 .

28. Siman JH, Forsgren A, Berglund G, Floren CH. Tobacco smoking increases the risk for gastric adenocarcinoma among Helicobacter pylori-infected individuals. Scand J Gastroenterol 2001;2;20813.

29. Zaridze D, Borisova E, Maximovitch D, Chkhikvadze V. Alcohol consumption, smoking and risk of gastric cancer: case-control study from Moscow, Russia. Cancer Causes Control 2000;11:36371.

30. Takahashi M, Nishikawa A, Furukawa F, Enami T, Hasegawa T, Hayashi Y. Dose-dependent promoting effects of sodium chloride $(\mathrm{NaCl})$ on rat glandular stomach carcinogenesis initiated with N-methyl-N'-nitro-N-nitrosoguanidine. Carcinogenesis 1994;7: 1429-32.

31. Nozaki K, Shimizu N, Inada K, Tsukamoto T, Inoue M, Kumagai $\mathrm{T}$, et al. Synergistic promoting effects of Helicobacter pylori infection and high salt diet on gastric carcinogenesis in Mongolian gerbils. Jpn J Cancer Res 2002;93:1083-9.

32. Takahashi M, Hasegawa R. Enhancing effects of dietary salt on both initiation and promotion stages of rat gastric carcinogenesis. Proc Princess Takamatsu Symp 1985;169-82.

33. Ji BT, Chow WH, Yang G, McLaughlin JK, Zheng W, Shu XO, et al. Dietary habits and stomach cancer in Shanghai, China. Int $\mathbf{J}$ Cancer 1998;76:659-64.

34. Tsugane S, Sasaki S, Kobayashi M, Tsubono Y, Akabane M. Validity and reproducibility of the self-administered food frequency questionnaire in the JPHC study group cohort I: study design, conduct and participant profiles. J Epidemiol 2003;13:S212.

35. Sasaki S, Kobayashi M, Ishihara J, Tsugane S. Self-administered food frequency questionnaire used in the 5-year follow-up survey of the JPHC study: questionnaire structure, computation algorithms, and area-based mean intake. J Epidemiol 2003;13:S1322.

36. Kobayashi M, Tsubono Y, Sasazuki S, Sasaki S. Tsugane S for the JPHC Study Group. Vegetables, fruit and risk of gastric cancer in Japan: a 10-year follow-up of the JPHC study cohort I. Int J Cancer 2002;102:39-44. 